\title{
PERDA DE TEMPERATURA EM REVESTIMENTOS ODONTOLÓGICOS, APÓS SUA REMOCÁO DO FORNO
}

\author{
Prof. Fernando Lyra Martins * \\ Prof. Léo Werner Süifert ** \\ Prof. Ennio Pessôa ***
}

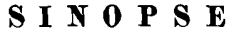

Verificou-se, comparativamente a perda de temperatura, após a remoça $\tilde{a}_{0}$ do forno, em quatro marcas comerciais de revestimentos odontológicos. Os ensaios de perda de temperatura foram efetuados em diferentes rolumes de revestimentos, em função dos espaços de tempo decorridos, ao serem retirados do forno a temperatura de 700 \%.

Decorridos dois minutos após a remoção, a perda de temperatura já atinge a ordem de 100 \% a 200:C.

\section{INTRODUCAO}

Nas técnicas rotineiras de fundições odontológicas, os cilindros contendo revestimento, em uma das etapas deste processo, são colocados no forno à temperatura ambiente, processando-se então, o aquecimento do mesmo até a temperatura de $700^{\circ}$ c.

Para que seja efetuada a fusão da liga de ouro e o seu subsequente vasamento, através de um canal de a- limentação previamente formado, para o interior do cilintro de revestimento, o cilindro em questão deve ser rapidamente removido do forno, momentos antes do vasamento propriamente dito.

Frequentemente, entre a remoção do cilindro de revestimento do forno e o vasamento da liga de ouro, decorrem espaços de tempo variáveis, dependendo dos conhecimentos e da habilidade do cirurgião-dentista.

Poucos autores se preocuparam com este assunto, entre os quais Crawford ${ }^{1}$, o qual afirma que «sendo o revestimento mau codutor de calor, a porção interior do cilindro é aquecida muito mais lentamente, do que a sua porção externa». O mesmo autor, no entanto, não nos oferece detalhes de como foram efetuados os ensaios de verificaçã desta diferença de temperatura.

Smith ${ }^{2}$ e Sourder et alii ${ }^{3}$, também se referem à perdas de temperaturas em revestimentos odontológicos, relacionando-as, inclusive à resistência à compressão. Não obstante os gráficos que apresentam, não nos

- Professor Auxiliar de Ensino de Materiais Dentários, Faculdade de Odontologia, UFRGN, Bolsa de Aperfeiçoamento de Ensino e Pesquisa, concedida pela CAPES.

* Professor Catedrático de Materiais Dentários, Faculdade de Odontologia da UFRGS.

*** Professor Adjunto de Materiais Dentários, Faculdade de Odontologia, UFRGS.

R. Fac. Odont. P. A.

13/14: 99-114, 1971/1972 
propiciam maiores detalhes de como foram efetuados os ensaios.

Yosso objetivo é o de verificar, em função dos espaços de tempos variáveis, acima referidos, qual a perda de calor que fatalmente ocorre na massa de revestimento aquecida, quando de sua remoção do forno.

c evidente que a perda de calor referida, está condicionada ao volume da massa de revestimento. Neste sentido, pretendemos estabelecer as CURVAS de perdas de calor em diversas marcas de revestimentos odontológicos, vasadas em diferentes tipos de cilindros para fundições, cilindros estes, protegidos ou não internamente com uma tira de asbesto e levados ao forno com ou sem a proteção externa de um cilindro metálico.

Eventualmente, poder-se-á alertar os cirurgiões-dentista, para a impor-

tância desta perda de calor, recomendando-se o tempo máximo que poderá decorrer entre a remoção do cilindro dorevestimento do forno e a execução da fundição propriamente dita.

\section{MATERIAIS E MÉTODOS}

\subsection{ELABORAÇ̃̃O DOS CORPOS DE PROVA}

Obtivems os corpos de prova, vasando as misturas de revestimentos, em sua consistência padrão, de acordo com as normas geralmente aceitas para este fim. ${ }^{4} e^{5}$

Como moldes para os corpos de prova, empregávamos cilindros metálicos ou cilindros de borracha, normalmente utilizados em inclusões normais para fundições odontológicas, os quais possuíam os seguintes volumes de capacidade:

VOLUME $\left(\mathrm{mm}^{3}\right)$

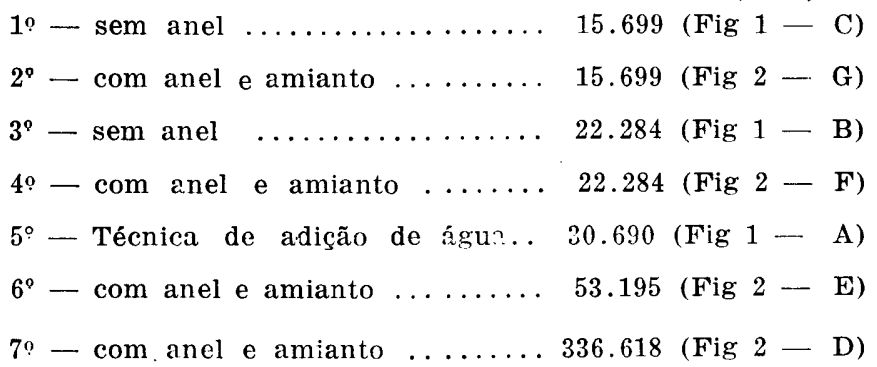

Fig 1 e Fig 2

Uma vez vasado o revestimento para o interior do cilindro metálico ou cilindro de borracha, inseria-se no centro geométrico da massa de revestimento, a ponta do terminal do par termo-elétrico. (Fig de N 3).
Nos casos de № 1,3 e 5 (sem anel) após decorridos 30 minutos do início da espatulação, liberava-se o corpo de prova dos cilindros metálicos ou do cilindro de borracha. 
Fig. 1

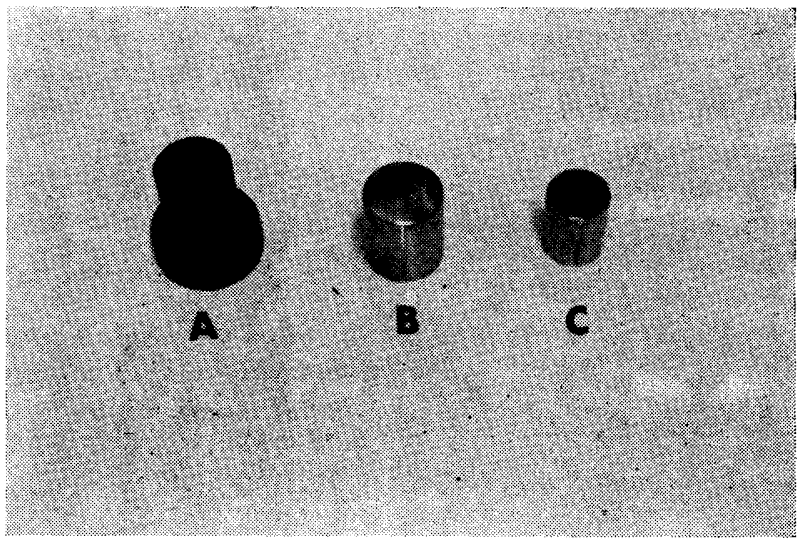

Fig. 2
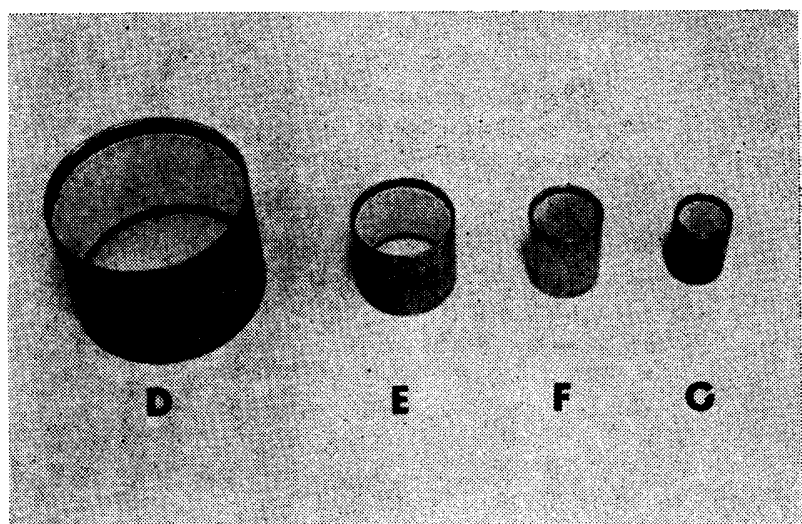

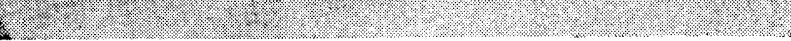




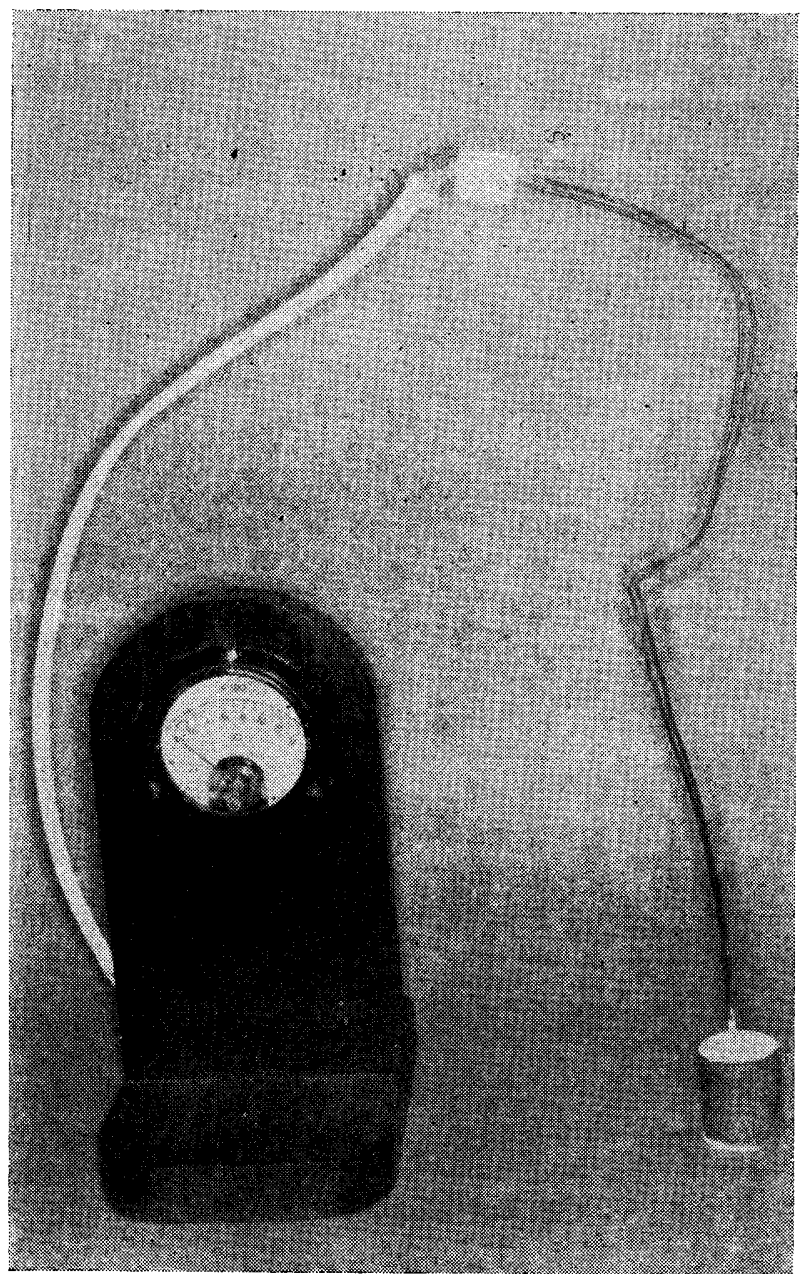

Fig. 3

R. Fac. Odont. P. A. 
Os revestimentos utilizados nesta pesquisa, bem como as proporções água/revestimento em que foram empregados, constam da Tabela de $\mathrm{N}^{\circ} 1$.

$\begin{array}{llllll}\text { T A B E L A } & \text { N } 0 & 1\end{array}$

\begin{tabular}{l|c|c|c|c}
\hline M A R C A & SIGLA & Proporcão A/P & FABRICANTE & $\begin{array}{c}\text { Parti- } \\
\text { da (No) }\end{array}$ \\
\hline HERODENT & A & 0,33 & Herman Josias S.A. & $\mathrm{n} / \mathrm{c}$ \\
\hline $\begin{array}{l}\text { EXCELSIOR } \\
\begin{array}{l}\text { EXPERIMENTAL } \\
\text { S. W. WHITE }\end{array}\end{array}$ & C & 0,35 & S.S. White Company & $\mathrm{n} / \mathrm{c}$ \\
\hline \begin{tabular}{l} 
CRISTOBALITE \\
\hline
\end{tabular} & D & 0,40 & S.S. White Company & $\mathrm{n} / \mathrm{c}$ \\
\hline
\end{tabular}

\subsection{ENSAIOS DE PERDA DE TEM.} PERATURA

Decorridas duas horas após o início da espatulação do revestimento, efetuávamos a ligação do terminal do par termo-elétrico «Cromel-Alumel», ao miliamperímetro, por intermédio de uma conexão de tarracha de parafusos.

Logo a seguir o corpo de prova era levado ao forno, que se encontrava à temperatura ambiente. Após ligarmos o forno, aguardávamos que o mesmo atingisse à temperatura de $700^{\circ} \mathrm{C}$. Desta maneira acompanháva- mo através do miliamperímetro, a elevação de temperatura do corpo de prova, no interior do forno.

Atingida a temperatura de $700^{\circ} \mathrm{C}$, retirava-se do forno, o corpo de prova e anotava-se as leituras de seu abaixamento de temperatura, através $d_{0}$ amperimetro, a espaços de tempo regulares e que eram os seguintes: $10,20,30,40,50$ e 60 segundos e, 2, $3,4,5,10,15$ e 20 minutos.

Os resultados obtidos porein ser observados nas Tabelas de No $2,3,4$ e 5, bem como nos Gráficos de N's I, II, II, IV, V, VI, VII e VIII. 


\section{3 - RESULTAdOS OBTIDOS}

TABELA 2

- REVESTIMENTO EXCELSIOR -

\begin{tabular}{|c|c|c|c|c|c|c|c|c|}
\hline \multirow{3}{*}{\multicolumn{2}{|c|}{ TEMPO }} & \multicolumn{7}{|c|}{ Perda de Temperatura dos Diversos Volumes a partir de $700^{\circ} \mathrm{C}$} \\
\hline & & \multirow[b]{2}{*}{$\begin{array}{l}15,699 \\
\text { s/anel }\end{array}$} & \multicolumn{3}{|c|}{ VOLUMES $\left(\mathrm{mm}^{3}\right)$ E } & \multicolumn{3}{|c|}{ CONDIÇÕES } \\
\hline & & & $\begin{array}{l}15,699 \\
\text { c/anel }\end{array}$ & $\begin{array}{l}22,284 \\
\text { s/anel }\end{array}$ & $\begin{array}{l}22,284 \\
\text { c/anel }\end{array}$ & $\begin{array}{l}30,690 \\
\text { s/anel }\end{array}$ & $\begin{array}{l}53,195 \\
\text { c/anel }\end{array}$ & $\begin{array}{r}336,618 \\
\text { c/anel } \\
\end{array}$ \\
\hline & $10 \mathrm{~s}$ & $690^{\circ}$ & $690^{\circ}$ & $690^{\circ}$ & $690^{\circ}$ & $690^{\circ}$ & $690^{\circ}$ & $690^{\circ}$ \\
\hline & $20 \mathrm{~s}$ & 680 & 690 & 690 & 690 & 690 & 680 & 690 \\
\hline & $30 \mathrm{~s}$ & 655 & 680 & 685 & 685 & 690 & 690 & 690 \\
\hline & $40 \mathrm{~s}$ & 640 & 665 & 675 & 675 & 680 & 650 & 680 \\
\hline & $50 \mathrm{~s}$ & 620 & 655 & 670 & 665 & 680 & 640 & 670 \\
\hline & $60 \mathrm{~s}$ & 605 & 645 & 660 & 655 & 670 & 625 & 670 \\
\hline & MIN. & 500 & 575 & 600 & 615 & 635 & 580 & 650 \\
\hline 3 & $"$ & 405 & 500 & 525 & 560 & 570 & 540 & 640 \\
\hline 4 & $"$ & 340 & 430 & 460 & 515 & 520 & 515 & 630 \\
\hline 5 & $"$ & 280 & 365 & 400 & 460 & 460 & 490 & 620 \\
\hline 10 & $"$ & 125 & 180 & 190 & 250 & 230 & 320 & 560 \\
\hline 15 & " & 60 & 110 & 100 & 150 & 130 & 210 & 510 \\
\hline 20 & $"$ & 30 & 70 & 60 & 90 & 70 & 140 & 450 \\
\hline
\end{tabular}

R. Fac. Odont. P. A. 
TABELA :

- REVESTIMENTO EXPERIMENTAL S. S. WHITE -

\begin{tabular}{|c|c|c|c|c|c|c|c|c|}
\hline \multirow{3}{*}{\multicolumn{2}{|c|}{ TEMPO }} & \multicolumn{7}{|c|}{ Perla de Temperatura dos Diversos Volumes a partir de $700^{\circ} \mathrm{C}$} \\
\hline & & \multirow[b]{2}{*}{$\begin{array}{l}15,699 \\
\text { s/anel }\end{array}$} & \multicolumn{3}{|c|}{ VOLUMES $\left(\mathrm{mm}^{3}\right) \mathrm{E}$} & \multicolumn{3}{|c|}{ CONDIÇÕES } \\
\hline & & & $\begin{array}{l}15,699 \\
\text { c/anel }\end{array}$ & $\begin{array}{l}22,284 \\
\text { s/anel }\end{array}$ & $\begin{array}{l}22,284 \\
\mathrm{c} / \text { anel }\end{array}$ & $\begin{array}{l}30,690 \\
\text { s/anel }\end{array}$ & $\begin{array}{r}53,195 \\
\text { c/anel } \\
\end{array}$ & $\begin{array}{r}336,618 \\
\text { c/anel } \\
\end{array}$ \\
\hline & $10 \mathrm{~s}$ & $690^{\circ}$ & $700^{\circ}$ & $690^{\circ}$ & $700^{\circ}$ & $700^{\circ}$ & $700^{\circ}$ & $690^{\circ}$ \\
\hline & $20 \mathrm{~s}$ & 680 & 690 & 680 & 690 & 690 & 690 & 690 \\
\hline & $30 \mathrm{~s}$ & 670 & 680 & 675 & 685 & 680 & 680 & 690 \\
\hline & $40 \mathrm{~s}$ & 660 & 670 & 670 & 680 & 675 & 670 & 690 \\
\hline & $50 \mathrm{~s}$ & 650 & 660 & 660 & 670 & 670 & 665 & 690 \\
\hline & $60 \mathrm{~s}$ & 640 & 650 & 650 & 660 & 665 & 660 & 690 \\
\hline & MIN. & 540 & 565 & 590 & 610 & 610 & 620 & 680 \\
\hline 3 & $"$ & 420 & 475 & 510 & 570 & 540 & 580 & 660 \\
\hline 4 & ” & 340 & 410 & 430 & 490 & 465 & 540 & 650 \\
\hline 5 & ” & 280 & 340 & 370 & 430 & 400 & 500 & 640 \\
\hline 10 & " & 125 & 170 & 185 & 230 & 200 & 320 & 590 \\
\hline 15 & $"$ & 60 & 90 & 90 & 140 & 110 & 200 & 530 \\
\hline 20 & , & 30 & 50 & 50 & 90 & 60 & 140 & 450 \\
\hline
\end{tabular}

R. Fac. Odont. P. A. 
TABELA 4

- REVESTIMENTO CRISTOBALITE -

\begin{tabular}{|c|c|c|c|c|c|c|c|c|}
\hline \multirow{3}{*}{\multicolumn{2}{|c|}{ TEMPO }} & \multicolumn{7}{|c|}{ Perda de Temperatura dos Diversos Voinmes a partir de $700^{\circ} \mathrm{C}$} \\
\hline & & \multirow[b]{2}{*}{$\begin{array}{l}15,699 \\
\text { s/anel }\end{array}$} & \multicolumn{3}{|c|}{ VOLUMES $\left(\mathrm{mm}^{3}\right)$ E } & \multicolumn{3}{|c|}{ CONDIÇÕES } \\
\hline & & & $\begin{array}{l}15,699 \\
\text { c/anel }\end{array}$ & $\begin{array}{l}22,284 \\
\text { s/anel }\end{array}$ & $\begin{array}{l}22,284 \\
\text { c/anel }\end{array}$ & $\begin{array}{l}30,690 \\
\text { s/anel }\end{array}$ & $\begin{array}{l}53,195 \\
\mathrm{c} / \text { anel }\end{array}$ & $\begin{array}{r}336,618 \\
\text { c/anel }\end{array}$ \\
\hline & $10 \mathrm{~s}$ & $700^{\circ}$ & $695^{\circ}$ & $700^{\circ}$ & $700^{\circ}$ & $695^{\circ}$ & $695^{\circ}$ & $690^{\circ}$ \\
\hline & $20 \mathrm{~s}$ & 685 & 680 & 685 & 690 & 685 & 680 & 690 \\
\hline & $30 \mathrm{~s}$ & 665 & 660 & 670 & 670 & 670 & 665 & 690 \\
\hline & $40 \mathrm{~s}$ & 650 & 640 & 660 & 660 & 640 & 650 & 690 \\
\hline & $50 \mathrm{~s}$ & 635 & 630 & 640 & 650 & 645 & 635 & 680 \\
\hline & $60 \mathrm{~s}$ & 615 & 610 & 630 & 640 & 630 & 625 & 660 \\
\hline & MIN. & 500 & 520 & 550 & 575 & 575 & 575 & 630 \\
\hline 3 & $"$ & 395 & 430 & 465 & 510 & 495 & 535 & 610 \\
\hline 4 & $"$ & 315 & 360 & 395 & 445 & 430 & 490 & 590 \\
\hline 5 & " & 260 & 300 & 325 & 385 & 365 & 450 & 570 \\
\hline 10 & " & 100 & 150 & 155 & 210 & 190 & 265 & 510 \\
\hline 15 & $"$ & 55 & 85 & 75 & 120 & 100 & 185 & 430 \\
\hline 20 & " & 25 & 50 & 45 & 80 & 65 & 115 & 350 \\
\hline
\end{tabular}

R. Fac. Odont. P. A. 
TABELA 5

- REVESTIMENTO HERODENT -

\begin{tabular}{|c|c|c|c|c|c|c|c|c|}
\hline \multirow{3}{*}{\multicolumn{2}{|c|}{ TEMPO }} & \multicolumn{3}{|c|}{ Perda de Temperatura dos } & diversos & \multirow{2}{*}{\multicolumn{2}{|c|}{$\begin{array}{l}\text { Volumes a } \\
\text { CONDIÇõES }\end{array}$}} & \multirow[t]{2}{*}{$700^{\circ} \mathrm{C}$} \\
\hline & & \multicolumn{4}{|c|}{ VOLUMES $\left(\mathrm{mm}^{3}\right) \mathrm{E}$} & & & \\
\hline & & $\begin{array}{l}15,699 \\
\text { s/anel }\end{array}$ & $\begin{array}{l}15,699 \\
\text { c/anel }\end{array}$ & $\begin{array}{l}22,284 \\
\text { s/anel }\end{array}$ & $\begin{array}{l}22 ; 284 \\
\text { c/anel }\end{array}$ & $\begin{array}{l}30,690 \\
\text { s/anel }\end{array}$ & $\begin{array}{l}53,195 \\
\text { c/anel }\end{array}$ & $\begin{array}{r}336,618 \\
\text { c/anel }\end{array}$ \\
\hline & $10 \mathrm{~s}$ & $690^{\circ}$ & $690^{\circ}$ & $690^{\circ}$ & $690^{\circ}$ & $690^{\circ}$ & $690^{\circ}$ & $690^{\circ}$ \\
\hline & $20 \mathrm{~s}$ & 670 & 680 & 690 & 680 & 690 & 665 & 690 \\
\hline & $30 \mathrm{~s}$ & 655 & 660 & 675 & 675 & 680 & 645 & 690 \\
\hline & $40 \mathrm{~s}$ & 625 & 640 & 665 & 665 & 670 & 635 & 690 \\
\hline & $50 \mathrm{~s}$ & 610 & 630 & 655 & 655 & 660 & 615 & 690 \\
\hline & $60 \mathrm{~s}$ & 590 & 610 & 645 & 645 & 650 & 605 & 685 \\
\hline & MIIN. & 485 & 515 & 585 & 595 & 605 & 550 & 670 \\
\hline 3 & $"$ & 390 & 440 & 500 & 535 & 535 & 515 & 655 \\
\hline 4 & 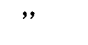 & 315 & 375 & 420 & 475 & 475 & 480 & 645 \\
\hline 5 & , & 260 & 325 & 350 & 415 & 405 & 440 & 635 \\
\hline 10 & $"$ & 100 & 170 & 150 & 215 & 190 & 275 & 575 \\
\hline 15 & $"$ & 50 & 85 & 75 & 120 & 95 & 170 & 515 \\
\hline 20 & $"$ & 30 & 55 & 45 & 70 & 50 & 110 & 435 \\
\hline
\end{tabular}

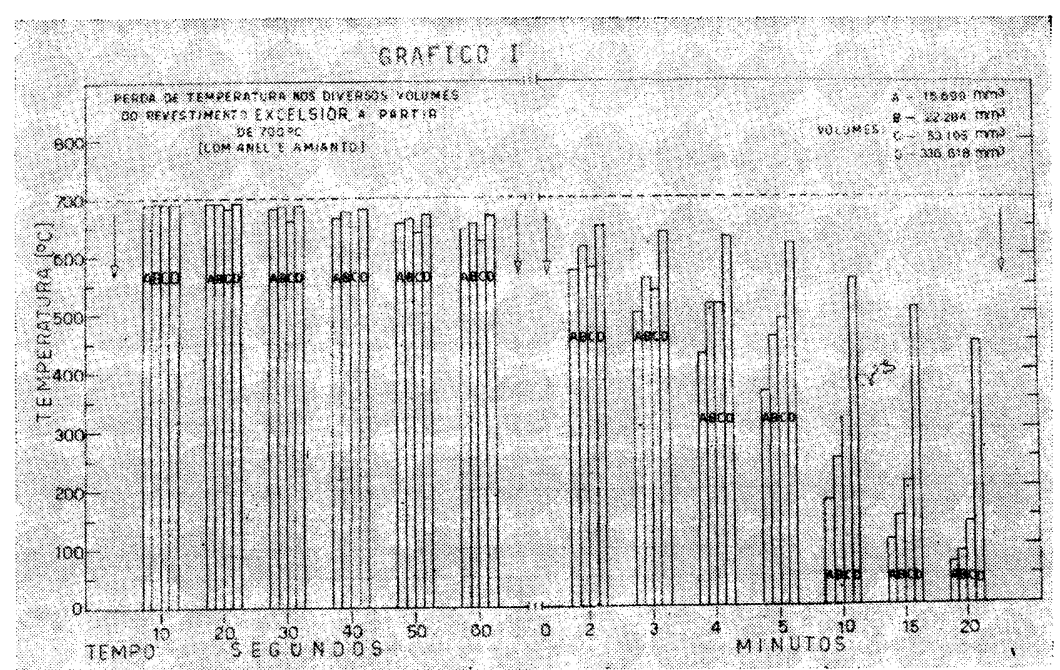

R. Fac. Odont. P. A. 



R. Fac. Odont. P. A. 

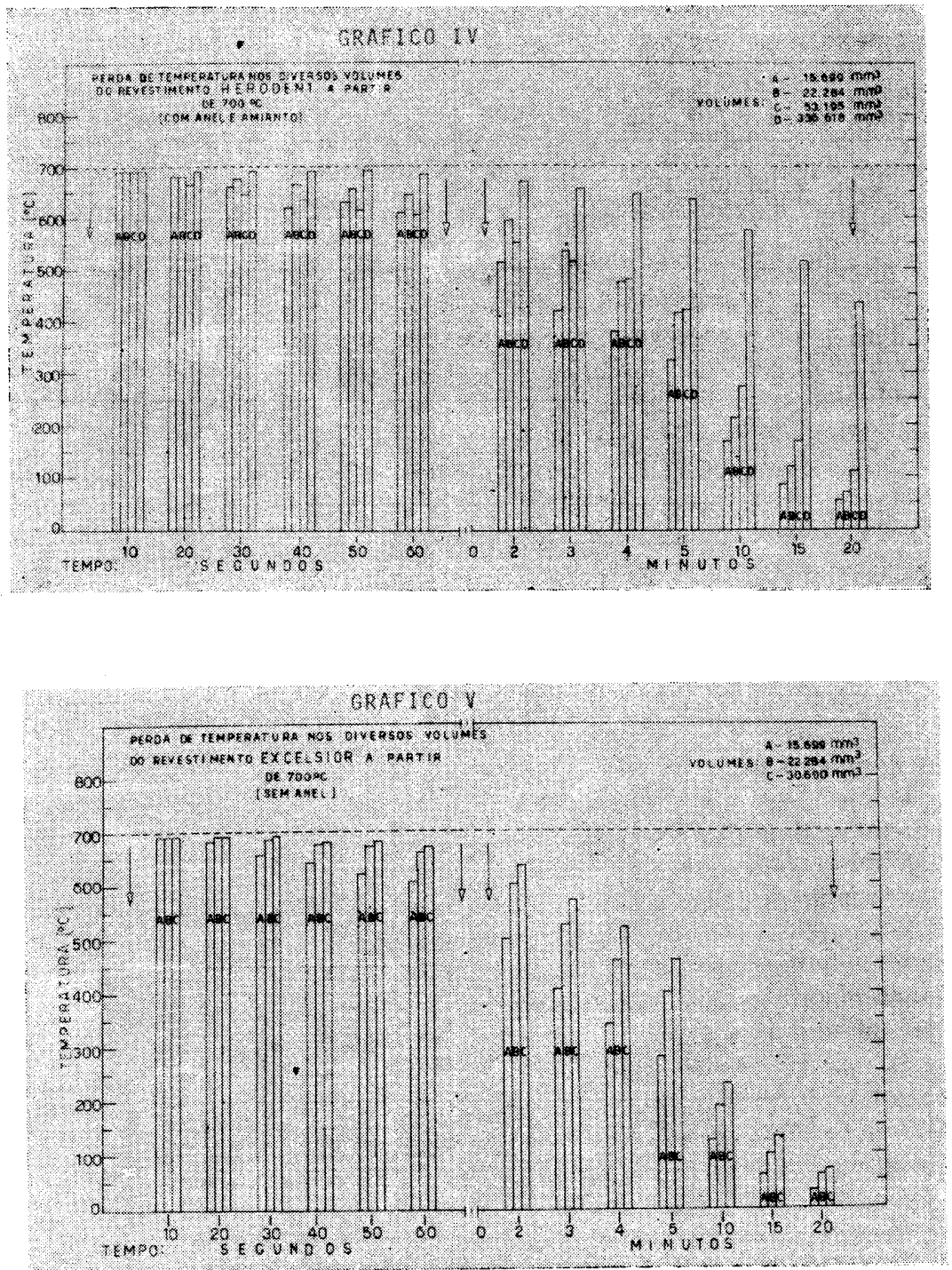

R. Fac. Odont. P. A.

13/14: 99-114, 1971/1972 

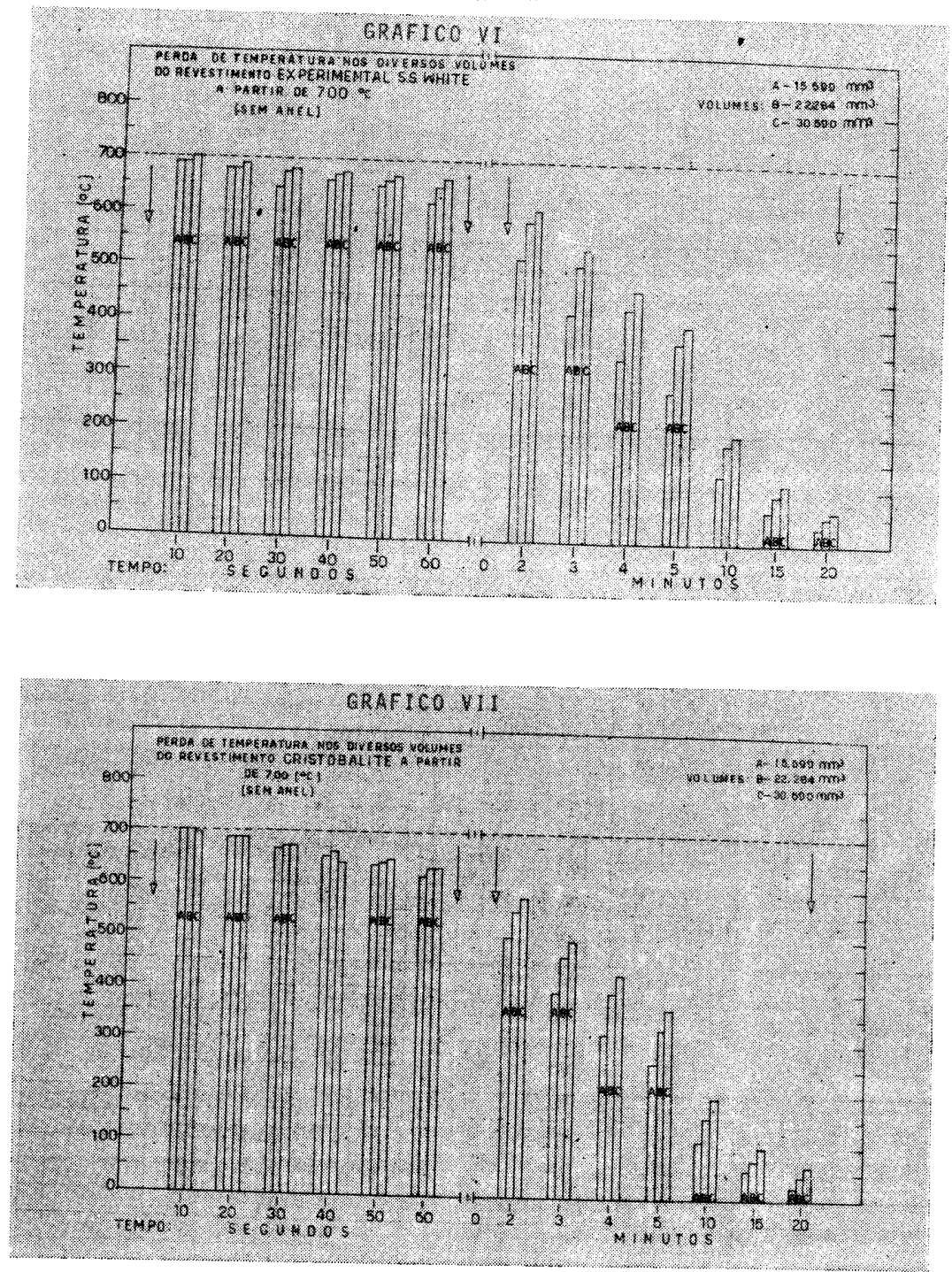

R. Fac. Odont P. A.

13/14: 99-114, 1971/1972 


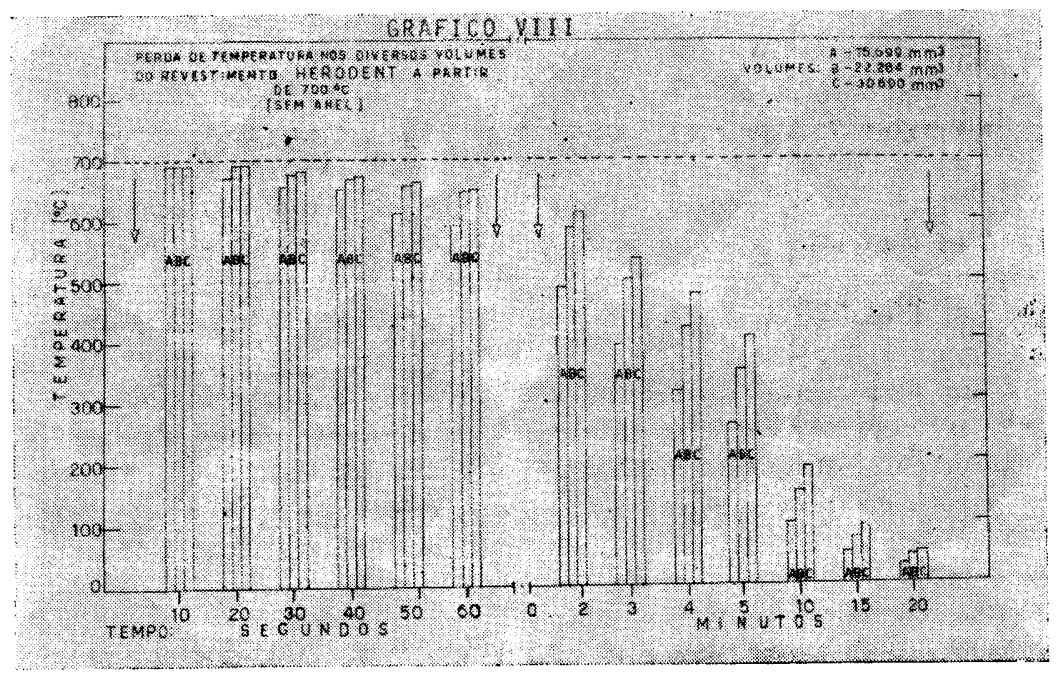

4. INTERPRETACÃO DOS RESULTADOS E CONCLUSÕES

\subsection{CILINDROS DE REVESTI-} MENTO COM ANEL E COM AMIANT0 (Gráficos I a IV)

\subsubsection{Até aos primeiros 60 segun-} dos, observa-se uma aparente discrepância no resfriamento. Há mais perda de calor nos cilindros «C» do que nos cilindros «B», apesar dos primeiros possuírem mais do que o dobro do volume. A explicação reside, em nosso entender, no fato de que os volumes «C» são os únicos contidos em um cilindro de latão, ao passo que os demais são contidos por um cilindro de aço inoxidável. Isto nos permite concluir que o material de que é constituído o cilindro, possue capital importância em relação à perda de calor do conjunto, nos primeiros 60 segundos.

4.1.2 $\overline{\mathrm{A}}$ partir do $4^{\mathrm{o}}$ minuto, para todos os revestimentos $\mathbf{e}, \mathrm{a}$ partir do $2^{\circ}$ minuto para os revestimentos Experimental S. White e Cristobalite da Kerr, a perda de calor é inversamente proporcional ao volume do material.

4.1.3 A importância da rapidez com que se deve proceder entre a remoção do cilindro de revestimento do forno e a execução da fundição propriamente dita ficou comprovada. A velocidade deve ser inversamente proporcional ao volume do revestimento contido no cilindro. 
4.2 CILINDROS DE REVESTIMENTO SEM ANEL (Gráfi$\cos \mathrm{V}$ a VIII)

4.2.1 Em todos os espaços de tempo decorridos após a remoção do forno e, para as 4 marcas de revéstimentos estudadas, a perda de calor é inversamente proporcional ao volume.

4.2.2 Ao atingirmos o $2^{\circ}$ minuto, a perda de temperatura já atinge à ordem de $100^{\circ} \mathrm{C}$ à $200^{\circ} \mathrm{C}$.

Esta última observação nos permite concluir que no caso de fundições or Técnicas de Expansão Higroscópica dos revestimentos, não deverão decorrer mais do que dois minutos entre a remoção do cilindro de revestimento do forno e a execução da fundição propriamente dita.

\section{RESUMO}

Frequentemente, entre a remoção do cilindro de revestimento do forno e o vasamento da liga metálica, decorrem espaços de tempo variáveis, condicionados à habilidade e aos conhecimentos do cirurgião-dentista. E evidente que este espaço de tempo decorrido é acompanhado de perda de calor por parte da massa de revestimento antes aquecida. Em 4 marcas de revestimento, comunmente empregadas pelo ci- rurgião-dentista brasileiro, vasados em diferentes tipos de cilindros para fundições, cilindros estes, protegidos ou não internamente com uma tira de asbesto e, levados ao forno com ou sem a proteção externa de um cilindro metálico, verificamos à espaços de tempo regulares a perda de temperatura. Os resultados são apresentados na forma de Tabelas e Gráficos, e dizem da importância de mais este detalhe técnico na execução de fundições odontológicas.

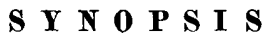

The time elepsed between the removal from the furnace of a casting cylinder and the casting proper, depends on the knowledge and ability of the operator. The heat loss, after removal of a furnace temperature of $700 \% \mathrm{C}$ at different time intervals, was measured in four dental casting investiments, using different volumes of investment, with or withont the protection of a casting ring and with or without asbestos liner. The results, presented in the form of tables and graphs, tell us about the importance of this additional technical detail, in the dental casting operation. Temperature losses in the order of from $100^{\circ}$ to $200^{\circ} \mathrm{C}$ were verified, already two minutes after removal from the furnace. 


\section{REFERÊNCIAS BIBLIOGRÁFICAS}

1. CRAWFORD, W.H. - Selection and use of investments, sprues, casting equipament and gold alloys in making small castings. JADA, 27:1459-70, Sep. 1940.

2. SMITH, Campbell J. - The chemistry and metallurgy of dental materials. 2.ed. London, Blackwell Scientific Publ., 1949, p.166.

3. SOUDER, W. \& PAFFENBARGER, G.C. - Physical properties of dental materials. Washington, National Bureau of Standards, 1942, p.88 Circular C433)

4. AMERICAN DENTAL ASSOCIATION, COUNCIL ON DENTAL MATERIALS AND DEVICES. Guide to dental materials and devices: 1968-60. 4.ed. Chicago, 1968, p-125-29.

5. GRUPO BRASILEIRO DE MATERIAIS DENTÁRIOS - Especificações brasileiras n? 11: especificações para revestimentos para fundições de liga de ouro odontológico. Porto Alegre, 1966, p.1-5 